\title{
Calcium Distribution during Anther Development in Oil Tea (Camellia oleifera Abel.)
}

\author{
Dongmei Wei \\ School of Life Science, Taizhou University, Jiaojiang, Zhejiang 318000, P.R. China \\ Chao Gao and Deyi Yuan' \\ Key Laboratory of Cultivation and Protection for Non-Wood Forest Trees of Ministry of Education \\ and the Key Laboratory of Non-Wood Forest Products of Forestry Ministry, Central South University \\ of Forestry and Technology, Changsha 410004, P.R. China
}

\begin{abstract}
AdDitional Index words. anther, development, calcium, Camellia oleifera, pollen
Abstract. The mechanism by which calcium regulates anther development remains unclear. This study investigated the relationship between calcium distribution and anther development in oil tea (Camellia oleifera Abel.) by using the potassium antimonite technique. Before the onset of microsporogenesis, abundant minute calcium precipitates appeared on the plasma membranes of microspore mother cells. Meanwhile, numerous precipitates accumulated in the tapetal cells. After meiosis, calcium precipitates appeared in young microspores. During microspore development, calcium precipitates mainly appeared in the small vacuoles of the cytoplasm. At the late microspore stage, a large vacuole formed, and the number of precipitates in the microspore decreased. The number of precipitates in the tapetal cells decreased as microsporogenesis proceeded. Then, calcium precipitates in the bicellular pollen cytoplasm again increased in number. During bicellular pollen development, the number of calcium precipitates decreased. As the pollen grains matured, only a few calcium precipitates were evident in the pollen cytoplasm. The results of this study, which show the spatial and temporal features of calcium distribution during the anther development of $C$. oleifera, suggest that calcium distribution is related to anther development.
\end{abstract}

Calcium is an essential element and an important ubiquitous messenger that participates or modulates many intracellular metabolic processes of plant growth and development (Bush, 1995; Ge et al., 2007a; Hepler and Wayne, 2005; Jones and Lunt, 1967). In flowering plants, calcium plays crucial roles in regulating sexual reproduction processes such as pollen germination, pollen tube growth (Cardenas et al., 2008; Franklin-Tong, 1999; Hepler, 1997; Heslop-Harrison, 1987; Kwack, 1967; Steinhorst and Kudla, 2013), and fertilization (Antonie et al., 1999; Digonnet et al., 1997; Santella et al., 2004; Tian and Russell, 1997; Whitaker, 2006). However, insufficient information is available on calcium distribution during microsporogenesis and anther development. The development of anthers is complicated. Cells in different anther tissues undergo different processes that lead to conspicuous changes in morphology and structure; these processes include meiosis in microspore mother cells, degeneration of tapetal cells, polarization in microspores during the formation of large vacuoles, and pollen wall formation after releasing from tetrads. Anther cell differentiation during anther development is strictly regulated in accordance with specific spatial and temporal processes, and calcium may play a role in some of these processes. Tian et al. (1998) found that numerous calcium precipitates accumulate within the tapetum and locules of Oryza sativa anthers during anther development. They also discovered that sterile anthers display abnormal calcium distribution compared with fertile anthers in a photoperiod-sensitive genetically male-sterile $O$. sativa line. Meanwhile, Meng et al. (2000) observed that more calcium

Received for publication 23 Sept. 2014. Accepted for publication 25 Nov. 2014. This work was supported by the Zhejiang Provincial Natural Science Foundation of China (No. Y3110395) and the National Natural Science Foundation of China (No. 31170639).

1Corresponding author. E-mail: qixin1981@hotmail.com; yuan-deyi@163.com. precipitates accumulate in the cells of the vascular bundles of anthers in a sterile Triticum aestivum line than in those of a fertile maintainer line. These results indicate that the abnormality of calcium distribution is related to anther abortion. However, to date, the features of calcium distribution in anthers have been investigated in only a few species (Ge et al., 2007b; Kong and Jia, 2004; Qiu et al., 2009), and the mechanism by which calcium regulates anther development is unclear. In the present study, we used potassium antimonite to localize and detect pools of loosely bound calcium in anther cells of $C$. oleifera, and we investigated the relationship between calcium distribution and anther development.

Camellia oleifera, a species of the Theaceae family, is native only to China. Camellia oil can be extracted from C. oleifera seeds, which have been used in China for more than 1000 years (Ruter, 2002). The polyunsaturated and good phenolic content of camellia oil is up to $80 \%$ (Yu et al., 1999). Camellia oil is a highquality cooking oil that is similar to olive oil and it stores well at room temperature (Ruter, 2002). However, the yield of $C$. oleifera is very low, which is closely related to sexual reproduction. Anther plays an important role in the sexual reproduction of $C$. oleifera. The present study aims to explore the role of calcium in anther development. This study may serve as a theoretical basis for the sexual reproduction of $C$. oleifera.

\section{Materials and Methods}

Camellia oleifera anthers selected at different developmental stages were squeezed and examined under a microscope to determine their developmental stages. The anthers were then fixed at room temperature for $3 \mathrm{~h}$ in $2 \%$ glutaraldehyde $(\mathrm{v} / \mathrm{v})$ in $0.1 \mathrm{~mol} \cdot \mathrm{L}^{-1}$ phosphate buffer $(\mathrm{pH} 7.8)$ that contains $1 \%$ 
potassium antimonite $\left(\mathrm{K}_{2} \mathrm{H}_{2} \mathrm{Sb}_{2} \mathrm{O}_{7} \cdot 4 \mathrm{H}_{2} \mathrm{O}\right)$. The use of potassium antimonite to precipitate calcium was detailed by Ge et al. (2007b). The samples were fixed in glutaraldehyde, washed three times for $30 \mathrm{~min}$ each in buffered $1 \%$ antimonite (w/v), and then postfixed in $1 \% \mathrm{OsO}_{4}(\mathrm{w} / \mathrm{v})$ containing $1 \%$ antimonite for $16 \mathrm{~h}$ at $4{ }^{\circ} \mathrm{C}$. The samples were then washed three times for $30 \mathrm{~min}$ each in buffer, dehydrated in a graded acetone series, and then embedded in Spurr's resin. At each stage, at least 10 anthers were embedded, and five samples were sectioned at $80 \mathrm{~nm}$ thick and stained with $2 \%$ uranyl acetate $(\mathrm{w} / \mathrm{v})$ in $50 \%$ methanol $(\mathrm{v} / \mathrm{v})$. Subsequently, the sections were then washed and air-dried before being observed and photographed under a transmission electron microscope (JEM-100CX; JEOL, Tokyo, Japan).

X-ray spectral analysis was performed under a scanning electron microscope (JSM-6390; JEOL). The peak deconvolution software INCA-Analyzer (Oxford Instruments, Oxford, U.K.) was used to analyze pollen grains, which were sliced $1 \mu \mathrm{m}$ thick and pasted on a glass slide.

\section{Results}

\section{Calcium distribution}

Microspore Mother CELl STAGE. Camellia oleifera has typical anthers with five layers of somatic cells in the anther wall, including one epidermal layer, one endothecium, two middle layers, and a tapetum. Before meiosis, the microspore mother cells (MMCs) separated from each other and were surrounded by thick callose walls (Fig. 1A). Numerous small organelles were observed in the cytoplasm of the MMCs. A layer of calcium-induced precipitates was observed on the callose walls of the MMCs and fewer precipitates in the cytoplasm (Fig. 1B).

Tetrad stage. After MMC meiosis, four haploid microspores were surrounded by a common callose wall to form a tetrad. Many precipitates remained on the surfaces of tetrad microspores. These precipitates were mainly limited to the pollen primexine, but a few were present in the microspore cytoplasm (Fig. 1C).

At this stage, some precipitates appeared in the tapetal cells of anther walls and connective tissue. When the tetrad callose wall dissolved, four microspores became free and began microspore development. Precipitates appeared in the free microspore cytoplasm and nucleus. However, the precipitates in the microspore cytoplasm did not exhibit specific distribution patterns at this stage (Fig. 1D).

At this developmental stage, the number of precipitates in the tapetal cells clearly decreased, and only a few were present in the small vacuoles of the cells. Many Ubisch bodies were

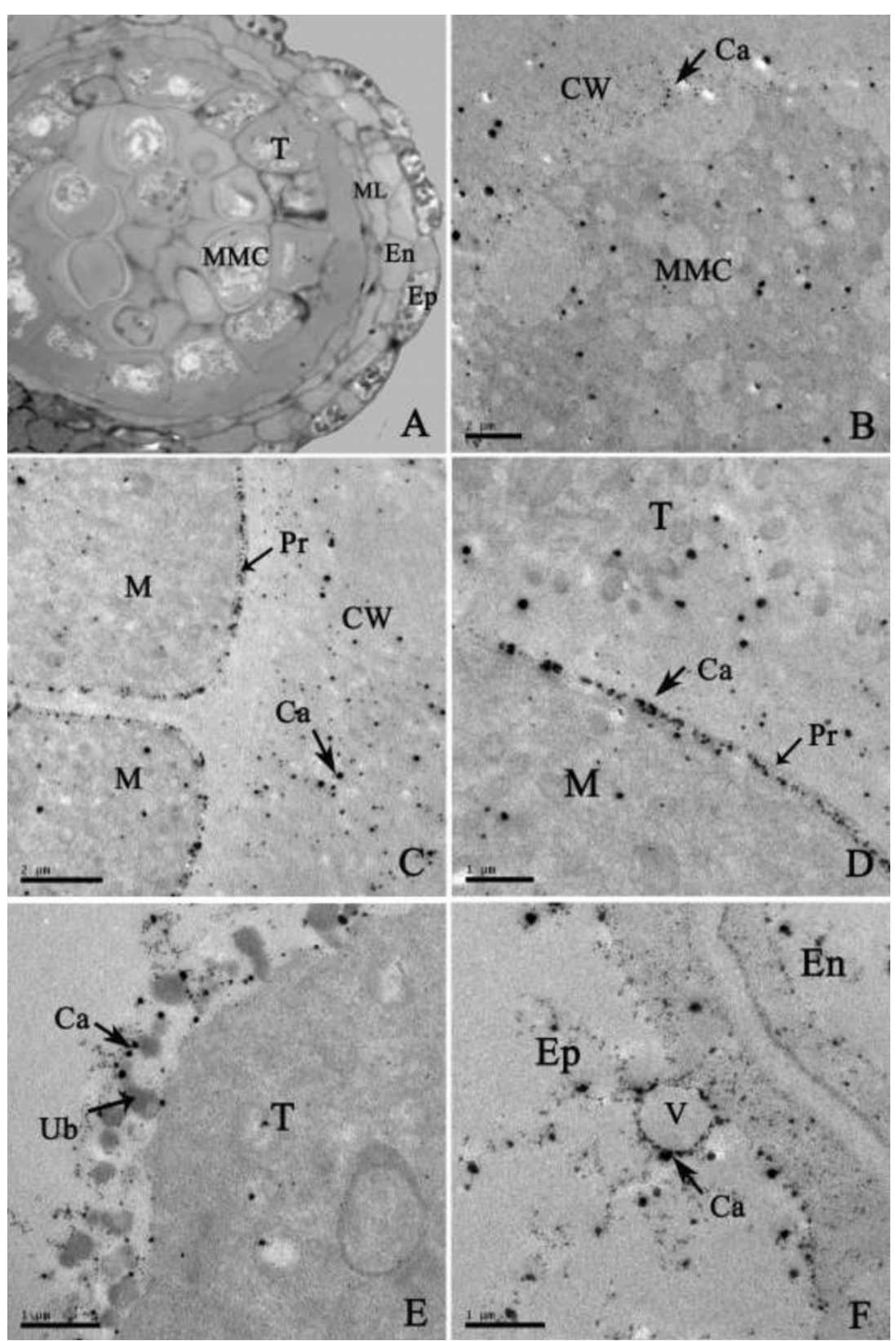

Fig. 1. Calcium precipitate distribution in young anthers of Camellia oleifera. (A) Transverse section at the microspore mother cells (MMCs) stage of a $C$. oleifera anther. (B) Numerous calcium precipitates accumulate on the callose wells of MMCs. (C) After MMC meiosis, numerous calcium precipitates remain on the surface of the tetrad microspore and in callose walls. (D) When the microspores are released from the tetrad, precipitates appear in the cells, particularly in the primexine. (E) The number of calcium precipitates in the tapetal cells decreases. (F) Numerous precipitates appear in the epidermal cells. $\mathrm{Ca}=$ calcium; $\mathrm{CW}=$ callose wall; $\mathrm{En}=$ endothecium; $\mathrm{Ep}=$ epidermis; $\mathrm{M}=$ microspore; $\mathrm{ML}=$ middle layer; $\mathrm{Pr}=$ primexine; $\mathrm{T}=$ tapetum; $\mathrm{Ub}=\mathrm{Ubisch}$ body $\mathrm{V}=$ vacuole. Bars: (A) $40 \mu \mathrm{m},(\mathbf{B}, \mathbf{C}) 2 \mu \mathrm{m},(\mathbf{D}, \mathbf{E}, \mathbf{F}) 1 \mu \mathrm{m}$. present on the surfaces of the tapetum (locules) and some of the precipitates were distributed on the external surface of the Ubisch bodies (Fig. 1E). In the epidermal cells of the anther wall, the number of calcium precipitates clearly increased and were mainly located in the vacuole membrane (Fig. 1F).

EARLY MICROSPORE STAGE. The early microspores contained a large nucleus in the center of the cell and no small vacuoles were evident in the cytoplasm. The calcium precipitates accumulated between the intine and the exine. After microspore 
development, some small vacuoles appeared in the cytoplasm and these vacuoles contained calcium precipitates (Fig. 2A). The number and size of vacuoles in the microspore increased and these vacuoles were distributed in the peripheral cytoplasm, which increased the number of calcium precipitates around the vacuoles (Fig. 2B).

LATE MICROSPORE STAGE. The small vacuoles fused to form large vacuoles, and the number of calcium precipitates decreased in the cytoplasmic matrix. The calcium precipitates were mainly located on the vacuolar membrane, but many also appeared on the microspore plasma membrane. The pollen intine and exine were found outside the membrane, and some precipitates were specifically located in the baculum of the exine (Fig. 2C). As the anthers underwent further development, small vacuoles in the microspores fused to form a large vacuole, which marked the late stage of microspore development. When the large vacuole formed, the microspore nucleus moved to the periphery and displayed unequal distribution. The number of calcium precipitates in the late microspores decreased, whereas the precipitates on the plasma membranes and in the exines completely disappeared (Fig. 2D).

At the late microspore stage, the cytoplasm matrix of anther epidermal cells still contained many calcium precipitates, but only a few were present in the endothecium and the middle cell layer (Fig. 2E). Meanwhile, the structure of the tapetal cells became indistinct and their cytoplasmic electron density increased, suggesting that the cells began to degenerate. The number of precipitates decreased in the degenerating tapetal cells (Fig. 2F).

To confirm that the calcium in late microspores dissolved into the large vacuole, we performed energy-dispersive X-ray spectral analysis of semithin sections of microspores (Fig. 3). Although the calcium precipitates at the late microspore stage disappeared, X-ray spectral analysis revealed that some calcium remained in the large vacuole (Fig. 4). This result suggested that calcium dissolved in the large vacuole.

Bicellular pollen grain Stage. After asymmetrical mitotic division of the microspore, a bicellular pollen grain was formed. This pollen grain consisted of a large vegetative cell and a small generative cell. At the early bicellular pollen stage, the large vacuoles in the vegetative cell disaggregated, and the density of the pollen cytoplasm increased. In the early bicellular pollen grains, the number of calcium precipitates increased again (Fig. 5A). This result suggested that the calcium that dissolved in the large vacuole of the vegetative cell returned to the cytoplasm after large vacuole

disaggregation. Then, the generative cell separated from the pollen intine, became spherical, and moved into the vegetative cell. The number of calcium precipitates observed between the generative and vegetative cells was the same (Fig. 5B).

At the early bicellular pollen stage, changes occurred in the anther wall cells; the calcium precipitates in the large vacuoles of the epidermal and endothecium cells became 


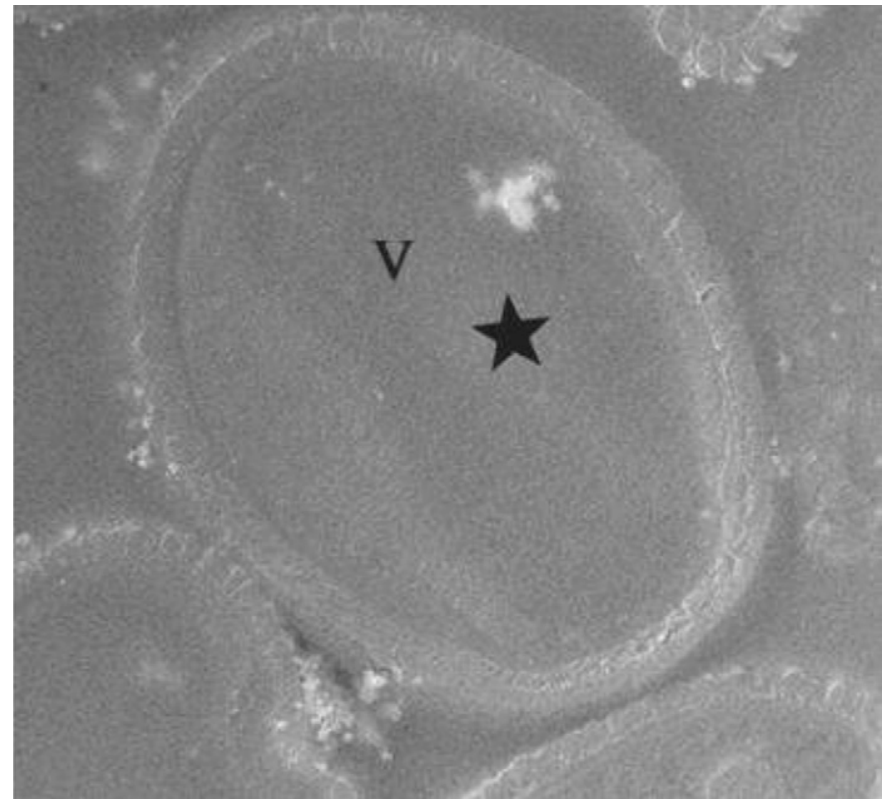

Fig. 3. The pollen grain with a large vacuole of Camellia oleifera at the microspore stage was sectioned (1 $\mu \mathrm{m}$ thick) and pasted on a slide. The asterisk indicates the large vacuole position in a microspore, which was analyzed by using an X-ray detector. $\mathrm{V}=$ vacuole.

smaller and formed abundant flocculent materials (Fig. 5C). The tapetal cells degenerated further and the cytoplasm matrix was reduced. No calcium precipitates accumulated in the degenerated tapetal cells (Fig. 5D). After pollen development, the generative cell inside the vegetative cell became elongated. Fewer calcium precipitates were present in the generative cell than in the vegetative cell (Fig. 5E). Near anthesis, only a few calcium precipitates were located in the small vacuoles in the mature pollen grains. However, many calcium precipitates were still present on the pollen surface (Fig. 5F).

\section{Discussion}

Before meiosis occurred in the MMCs of C. oleifera, the anthers accumulated numerous calcium precipitates, which were mainly located in the callose wall of the MMCs. During intine formation, calcium began to appear in the intine at the tetrad stage. After young microspores were released from the tetrad, calcium appeared between the endexine and the intine. At the mature pollen stage, a large number of calcium precipitates was present on the pollen exine. The pollen wall began to form at the tetrad stage. Previous studies showed that the cell wall is one of the largest calcium stores in plant cells and that pollen walls contain the most calcium in pollen grains (Kong and Jia, 2004). Furthermore, the pollen of Gasteria verrucosa (Tirlapur and Willemse, 1992) and Lilium longiflorum (Reiss et al., 1985) can release calcium during the hydration and early germination phases. Thus, we proposed that the accumulation of calcium in the callose walls of MMCs and the tetrad might be correlated with the formation of pollen walls. Moreover, the mature pollen wall might be a storage of calcium for pollen-pistil interaction and pollen germination.

In this study, there is an interesting phenomenon. The calcium precipitates were present in the cytoplasm of young microspores, particularly within small vacuoles. Then, the

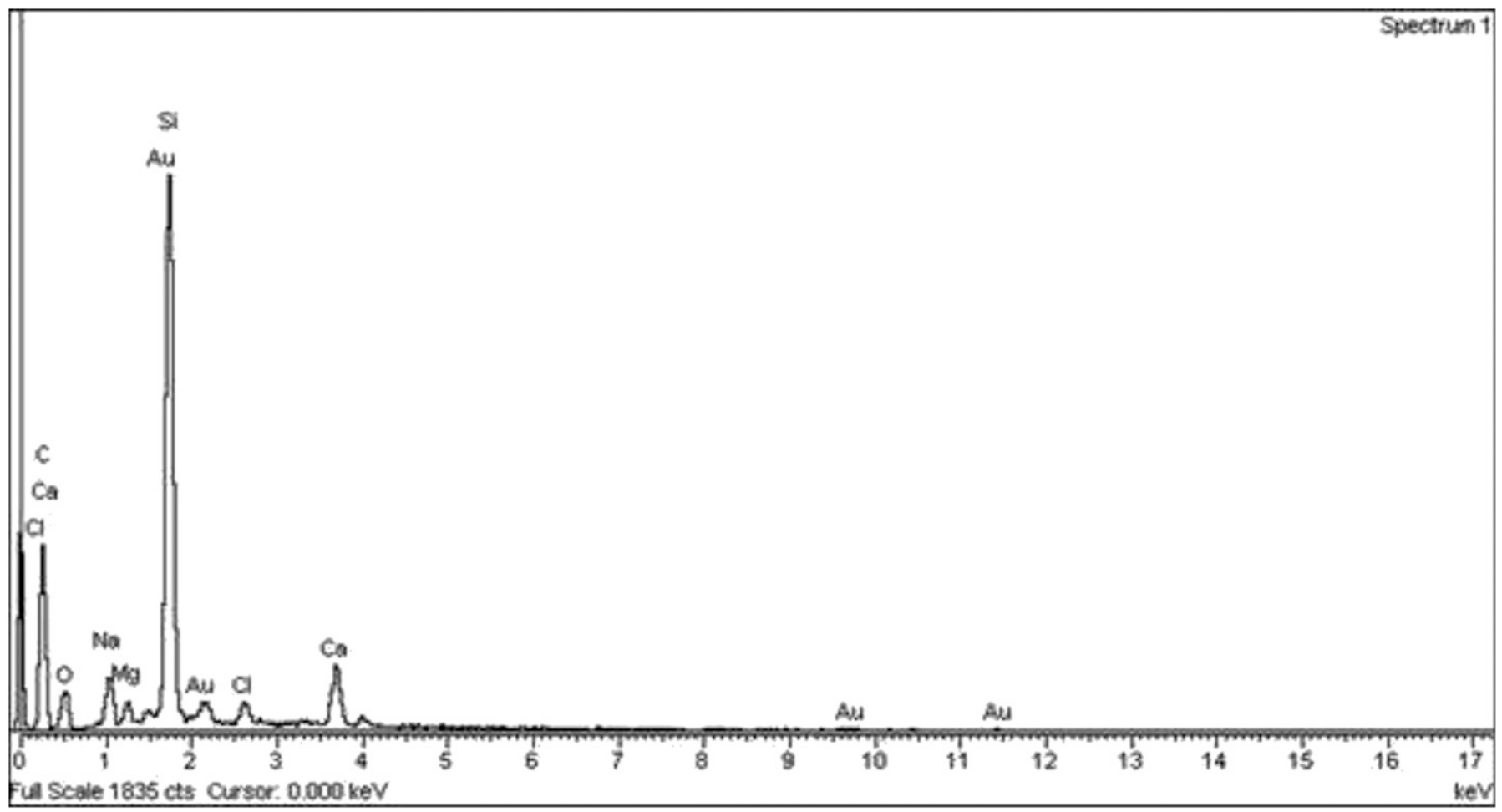

Fig. 4. Energy-dispersive X-ray spectra of semithin sections of large vacuoles in microspores of Camellia oleifera microspores. Calcium was present in the large vacuole. 

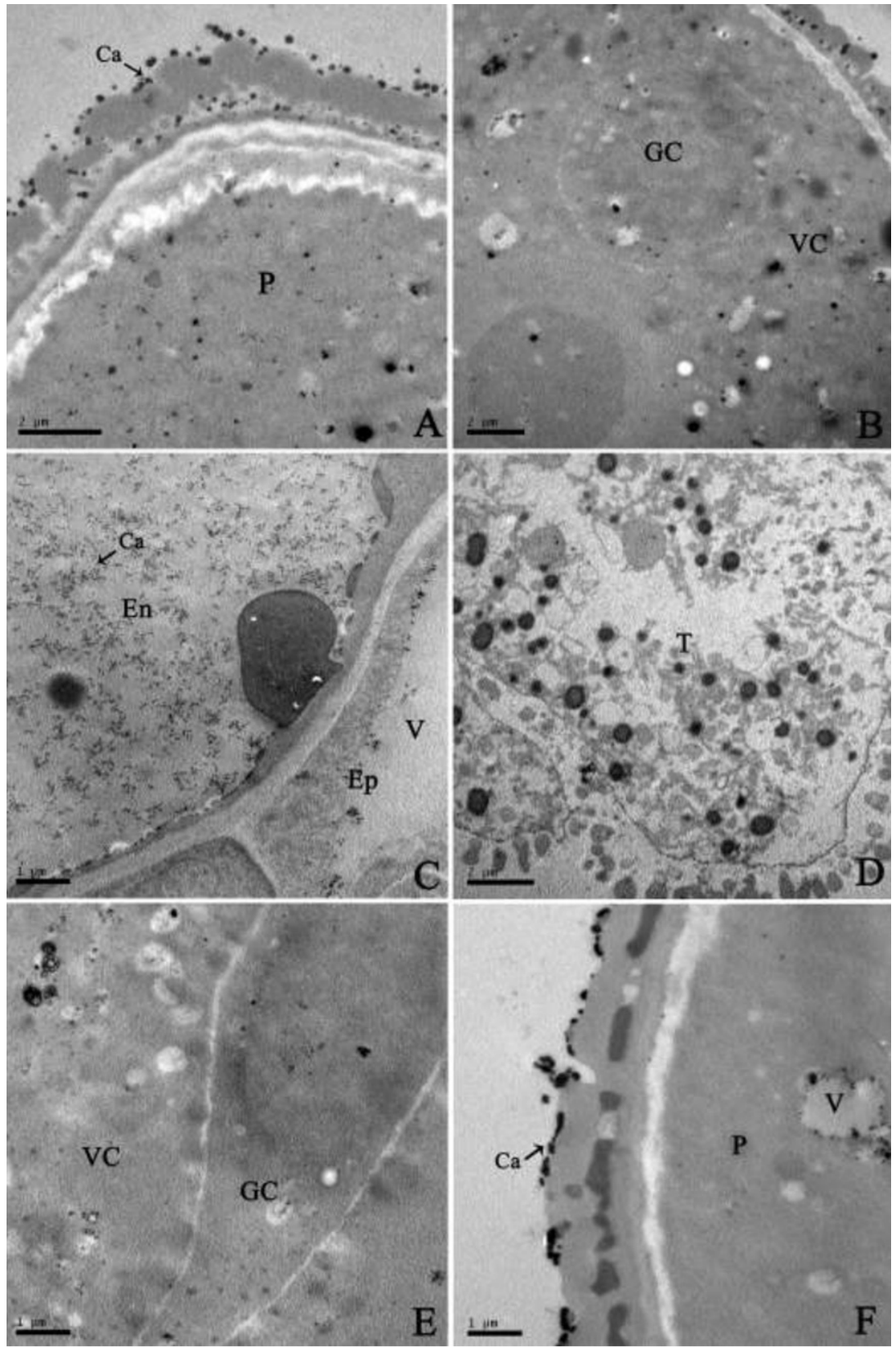

Fig. 5. Calcium precipitate distribution in Camellia oleifera anthers at the bicellular pollen stage. (A) After microspore division, the calcium precipitates in bicellular pollen increase in number again. (B) A few precipitates are present in the generative cells of the bicellular pollen. (C) At this time, calcium precipitates in the large vacuoles of epidermal cells become quite small. The endothecium cells produce large vacuoles, which contain some precipitates. (D) The tapetal cells degenerate, and no calcium precipitates are present in the cells. (E) In a mature pollen grain, a generative cell becomes elongated. Fewer precipitates are present in the generative cell than in the vegetative cell. (F) Numerous calcium precipitates accumulate on the surface of the mature pollen grain. En = endothecium; Ep = epidermis; $\mathrm{Ca}=$ calcium; $\mathrm{GC}=$ generative cell; $\mathrm{P}=$ pollen; $\mathrm{T}=$ tapetum; $\mathrm{V}=$ vacuole; $\mathrm{VC}=$ vegetative cell. Bars: $(\mathbf{A}, \mathbf{B}, \mathbf{D})$ $2 \mu \mathrm{m},(\mathbf{C}, \mathbf{E}, \mathbf{F}) 1 \mu \mathrm{m}$.

small vacuoles coalesced to form a large vacuole and the calcium precipitates disappeared from the late microspores. Hepler and Callaham (1987), Hepler and Wayne (1985), Izant (1983), and Wick et al. (1985) demonstrated that calcium is associated with critical events of mitosis in plant cells. After the microspore divided to produce a bicellular pollen grain, the large vacuole in the vegetative cell disaggregated and calcium precipitates reappeared in the vegetative cell cytoplasm. Then, the number of calcium precipitates in the bicellular pollen grains continued to decrease. Calcium is involved in the cell division and polarized tip growth process of pollen tubes (Tirlapur and Willemse, 1992). The calcium distribution pattern observed during $C$. oleifera anther development exhibited spatialtemporal features; high levels of calcium appeared in C. oleifera anthers at a specific time (at the microspore stage) and in a specific region (vacuole formation of microspores). This result suggests that calcium regulates large vacuole formation in microspores during pollen development in C. oleifera.

The physiological function of calcium during anther development remains unknown. Calcium can regulate many cellular functions according to its cellular location, binding, and solubility (Hepler and Wayne, 2005). Calcium also appears to play a unique and important role in plant cells during wall accretion, vacuolar turgor maintenance, and stomatal movement (Bolwell, 1993; De Silva et al., 1985a, 1985b; Inoue and Katoh, 1987; Schwartz et al., 1988). However, a few studies have examined calcium distribution during anther development and the physiological function of calcium in anther development has not previously been proposed. In the present study, calcium precipitates in anthers mostly accumulated during microspore development. Thus, the presumed functions of calcium in pollen development are as follows: 1) regulating the activation of voltage-dependent ion channels (Hedrich and Neher, 1987); and/or 2) providing osmotic pressure to aid in the formation of vacuoles within a microspore, which contributes to the polarization of the cytoplasm preceding the unequal division of the microspore. In this study, we presumed that the physiological function of calcium during anther development is regulating large vacuole formation in the microspore, which distributes microspore nuclei unequally and initiates microgametogenesis. As pollen matures, calcium leaves the pollen grain and accumulates on the pollen surface, which prepares the pollen for germination when it lands on a stigma.

\section{Literature Cited}

Antonie, A.F., J.E. Faure, S. Cordeiro, C. Dumas, M. Rougier, and J.A. Feijo. 1999. A calcium influx is triggered and propagates in the zygote as a wavefront during in vitro fertilization of flowering plants. Proc. Natl. Acad. Sci. USA 97:10643-10648.

Bolwell, G.P. 1993. Dynamic aspects of the plant extracellular matrix. Intl. Rev. Cytol. 146:261-324. 
Bush, D.S. 1995. Calcium regulation in plant cells and its role in signaling. Annu. Rev. Plant. Physiol. Mol. Biol. 46:95-122.

Cardenas, L., A. Lovy-Wheeler, J.G. Kunkel, and P.K. Hepler. 2008. Pollen tube growth oscillations and intracellular calcium levels are reversibly modulated by actin polymerization. Plant Physiol. 146:1611-1621.

De Silva, D.L.R., R.C. Cox, and A.M. Hetherington. 1985a. Suggested involvement of calcium and calmodulin in the responses of stomata to abscisic acid. New Phytol. 104:41-51.

De Silva, D.L.R., A.M. Hetherington, and T.A. Mansfield. 1985b. Synergism between calcium ions and abscisic acid in preventing stomatal opening. New Phytol. 100:473-482.

Digonnet, C., D. Aldon, N. Leduc, C. Dumas, and M. Rougier. 1997. First evidence of a calcium transient in flowering plants at fertilization. Development 124:2867-2874.

Franklin-Tong, V.E. 1999. Signaling and the modulation of pollen tube growth. Plant Cell 11:727-738.

Ge, L.L., H.Q. Tian, and S.D. Russell. 2007a. Calcium function and distribution during fertilization in angiosperms. Amer. J. Bot. 94:1046-1060.

Ge, L.L., C.T. Xie, D.M. Wei, Y.L. Qiu, and H.Q. Tian. 2007b. Calcium distribution during the anther development of tobacco. Intl. J. Dev. Biol. 1:287-293.

Hedrich, R. and E. Neher. 1987. Cytoplasmic calcium regulates voltage-dependent ion channels in plant vacuoles. Nature 329:833836.

Hepler, P.K. 1997. Tip growth in pollen tubes: Calcium leads the way. Trends Plant Sci. 2:79-80.

Hepler, P.K. and D.A. Callaham. 1987. Free calcium increases during anaphase in stamen hair cells of Tradeseantia. J. Cell Biol. 105:21372143.

Hepler, P.K. and R.O. Wayne. 1985. Calcium and plant development. Annu. Rev. Plant Physiol. 36:397-439.

Hepler, P.K. and R.O. Wayne. 2005. Calcium: A central regulator of plant growth and development. Plant Cell 17:2142-2155.

Heslop-Harrison, J. 1987. Pollen germination and pollen tube growth. Intl. Rev. Cytol. 107:1-78.

Inoue, H. and Y. Katoh. 1987. Calcium inhibits ion-stimulated stomatal opening in epidermal strips of Commelina communis L. J. Expt. Bot. 38:142-149.

Izant, J.G. 1983. The role of calcium ions during mitosis. Calcium participates in the anaphase trigger. Chromosoma 88:1-10.

Jones, R.G.W. and O.R. Lunt. 1967. The function of calcium in plants. Bot. Rev. 33:407-427.
Kong, H.Y. and G.X. Jia. 2004. Calcium distribution during pollen development of Larix principis-rupprechtii. Acta Biochim. Sinica 46:69-76.

Kwack, B.H. 1967. Studies on cellular site of calcium action in promoting pollen tube growth. Plant Physiol. 20:825-833.

Meng, X.H., J.B. Wang, and R.Q. Li. 2000. Effect of photoperiod on calcium distribution in photoperiod-sensitive cytoplasmic malesterile wheat during anther development. Acta Biochim. Sinica 42:15-22.

Qiu, Y.L., R.S. Liu, D.M. Wei, and H.Q. Tian. 2009. Calcium distribution in developing anther of lettuce (Lactuca sativa). Ann. Bot. Fenn. 46:101-106.

Reiss, H.D., W. Herth, and R. Nobiling. 1985. Development of membrane- and calcium-gradients during pollen germination of Lilium longiflorum. Planta 163:84-90.

Ruter, J.M. 2002. Nursery production of tea oil camellia under different light levels, p. 222-224. In: Janick, J. and A. Whipkey (eds.). Trends in new crops, new uses. ASHS Press, Alexandria, VA. Santella, L., D. Lim, and F. Moccia. 2004. Calcium and fertilization: The beginning of life. Trends Biochem. Sci. 29:400-408.

Schwartz, A., N. Ilan, and D.A. Grantz. 1988. Calcium effects on stomatal movement in Commelina communis L. Plant Physiol. 87:583-587.

Steinhorst, L. and J. Kudla. 2013. Calcium-A central regulator of pollen germination and tube growth. Biochim. Biophys. Acta 1833:1573-1581.

Tian, H.Q., A. Kuang, M.E. Musgrave, and S.D. Russell. 1998. Calcium distribution in fertile and sterile anthers of a photoperiodsensitive genetic male-sterile rice. Planta 204:183-192.

Tian, H.Q. and S.D. Russell. 1997. Calcium distribution in fertilized and unfertilized ovules and embryo sacs of Nicotiana tabacum L. Planta 202:93-105.

Tirlapur, U.K. and M.T.M. Willemse. 1992. Changes in calcium and calmodulin levels during microsporogenesis, pollen development and germination in Gasteria verrucosa (Mill.) H. Duval. Sex. Plant Reprod. 5:214-223.

Whitaker, M. 2006. Calcium at fertilization and in early development. Physiol. Rev. 86:25-88.

Wick, S.M., S. Muto, and J. Duniec. 1985. Double immuno-fluorescence labeling of calmodulin and tubulin in dividing plant cells. Protoplasma 126:198-206.

Yu, Y.S., S.X. Ren, and K.Y. Tan. 1999. Study on climatic regionalization and layer and belt distribution of oil tea camellia quality in china. J. Asian Nat. Prod. Res. 14:123-127. 